\title{
A Literature Review on Machine Vision based Approaches for Ripeness Detection of Fruits
}

\author{
Rencheeraj Mohan \\ Department of Computer Science \\ College of Engineering Poonjar \\ Kerala, India
}

\author{
Sreekumar K. \\ Department of Computer Science \\ College of Engineering Poonjar \\ Kerala, India
}

\begin{abstract}
Plump fruits are a vital part of the human diet giving mandatory vitamins, minerals and other health encouraging compounds. Quality assessment and finding of fruit ripeness is a major concern in agriculture business and becomes a growing research concern in computer vision. Image processing is an advanced field which led to a higher demand to reduce the high rate of errors and given more possible results. Therefore, the objective of many types of research is to standardize and reduce manual work in the classification of tomatoes ripeness. One of the most important feature of an image is color. Estimating the ripeness of fruits via color can be performed as it is the dominant feature in describing the information of the image. However, each color models have been given a different performance when used in the experiment. This paper is a survey of different techniques that are deployed over different varieties of fruit images in order to detect maturity stages for ripening, fruit region estimation and also, the effect of different color models and other features on detecting ripeness was studied in this literature survey.
\end{abstract}

\section{General Terms}

Computer Vision, Image Analysis, Machine Learning, Literature Survey, Pattern Analysis.

\section{Keywords}

Fruit classification, maturity detection, segmentation, classification, feature extraction. Ripeness detection.

\section{INTRODUCTION}

The production rate of each fruit in this world is increasing every year. Identifying and ripening of each fruit, based on their maturity stage is a difficult process. The standard of ripe fruit (such as texture, color, shelf life, sugar content) has an outstanding effect of impacts on consumer approving, resistance against pathogens and transportability to long distances. In earlier time fruit quality was examined by human vision. However, due to distraction, illness and other influential factors, there is a high error rate that occurred during working for a long duration. To minimize the failure rate humans should invent new inventions. It is important to deliver fruits and vegetables in high quality. A fast and costeffective method has to be invented without damage due to the high demand in many areas.

In recent years, by using computer vision technology many research was done on fruit quality grade. One of these ways is segmentation, which means to divide an image into various sections that are homogeneous with regard to some feature of an image. Human visual perception has an advanced image segmentation technique that can classify an object. Humans have utilized their sense of the vision to classify their environment from its surrounds in order to identify them.
Better image processing algorithms have to be implemented in order to process automatically the fruit defect and maturity detection. To develop an algorithm for image processing certain process are there: 1) to identify defect and maturity detection of fruits, and 2) test and verify the analysis of image processing with experimental results. The first step of an image analysis process is the image segmentation method. After the image segmentation, the features are extracted and shape is detected depends on the quality of the segmentation. Without an ideal segmentation algorithm, the target cannot be recognized. Moreover, the segmentation process decides the success or failure of the analysis.

\section{REVIEW OF EXISTING METHODS}

In this paper, $\mathrm{Wu}$ et al. [1] proposed a novel automatic algorithm for recognizing tomatoes for ripening by the help of an improved method that merges multiple features, feature analysis and selection, a weighted relevance vector machine (RVM classifier) and bi-layer classifier. The algorithm undergoes two levels of classification. The first layer of classification uses color difference information to detect regions containing tomatoes. Second classification strategy uses multi-medium features for training the classifier. Both operations are done separately, thus they avoid the errors from each strategy.

Images were acquired using a live metal oxide Semiconductor camera. A total of 120 images were randomly selected which are taken under natural daylight conditions (07:00-16:00, sunny days). Under complex background and light conditions, images were captured at $30-70 \mathrm{~cm}$ distance. A total of 120 images of resolution $4032 \times 3024$ pixels were obtained. Among these 60 belongs to sunny side images and other 60 belongs to shadow side images. 30 images were selected randomly as training set and rest 90 were used for testing.

The processed images were divided into $9 \mathrm{x} 9$ pixel blocks for simplifying the feature calculation and enhancing the recognition efficiency thus, improving the operating speed and computing efficiency. The main advantages of these blocks were in reducing the noise in feature vectors and reduction in computing time. Each pixel region has different weights. Regions were obtained by using the RGB color space. Features were extracted during the training and testing steps by using these blocks. Five texture-related features (entropy, Energy, Correlation, inertial moments and local smoothening) and six color-related features (Red, Green, Blue, Hue, Saturation, and Intensity) were extracted from each pixel block. These features were used to train the RVM classifier and to calculate the weight vector. RVM machine learning technique uses Bayesian inference to calculate probabilistic predictions and image blocks were classified using these features. Iterative RELIEF (I-RELIEF) algorithm was used to examine the relevant features and their weights to 
improve RVM classifier. I-RELIEF algorithm was used to determine the contribution ratios of the extracted color features and texture features for selecting the most relevant features among them and for determining the weight coefficients of the RVM classifier. Final results were obtained by combining the block classification results and the bi-layer classification strategy. The proposed algorithm had an accuracy of $94.90 \%$ for recognizing the tomato from 120 images and the recognition process took $2.94 \mathrm{~s}$ on the average. Due to the vast amount of data, the recognition time is quite long. For reducing cost the volume of the images taken should be reduced. For a real-world application, the identification times is too short.

The main objective of Tan's et al. [2] work was to develop an efficient computer vision method to identify the maturity of the blueberry fruit with three different growth stages: mature, intermediate and young using outdoor color images acquired from a commercial field. A total of 321 images (3648x2736) were captured between 10:00 AM and 12:00 PM local time using digital SLR camera on a sunny day with low wind and a few clouds in the sky. Blueberry detection was challenging due to complex background objects like leaves, burgundy and green branches, and soil.

HOG features and color features were used for recognizing and counting blueberry fruits of different maturity stages. The algorithm first uses HOG (Histogram Oriented Gradients) feature vectors to construct fruit training set using 1374 positive images and 2290 negative images which were cropped from the original color image and the algorithm computes the occurrences of gradient orientation in localized regions of an image with a sliding window. For extracting HOG features actual images were divided into smaller regions called cells, and for each pixel in each cell, a histogram of gradient directions was assembled together. Later the histograms were combined. Moreover, to improve the accuracy of each cell, intensity across a large region of the image was calculated and then using these values normalization was done for each cell within the larger regions called blocks. The size of each object in the blueberry images was different. Thus, the image should be searched using multiscale detection and this can be done through implementing a Gaussian pyramid of the image.

HOG feature descriptors couldn't categorize the growth stages of blueberry fruit. In order to achieve this color features should be extracted. The color features were extracted using 2290 patches cropped from the same original images. A linear SVM (Support Vector Machines) classifier was used to train the patches for detecting fruit like regions and SVM quickly clarify fruit objects from the background using HOG features. Non fruit regions were eliminated using $a^{*}$ and $b^{*}$ features in the $\mathrm{L}^{*} \mathrm{a} * \mathrm{~b}$ color space features. Different maturity stages were identified using the KNN (K-Nearest Neighbor) and TMWE (Template Matching with Weighted Euclidean Distance) classifiers. The output of the SVM classifier (images with HOG detection result) was given as the input to $\mathrm{KNN}$ and TMWE classifier.

KNN classifier obtained the best average accuracy of $86.0 \%$, $94.2 \%$ and $96.0 \%$ for young fruit, intermediate fruit, and mature fruit, respectively. KNN yielded an average accuracy of $96.1 \%$ and $95.1 \%$ for TMWE. False positive rate $3.3 \%$ for KNN and $4.1 \%$ for TMWE) and miss rate (5.2\% for KNN and $5.8 \%$ for TMWE) for mature fruit. For intermediate fruit average accuracy (94.2\% for KNN and $92.4 \%$ for TMWE), false positive rate of $4.6 \%$ and miss rate $(5.4 \%$ for $\mathrm{KNN}$ and $6.2 \%$ for TMWE). For young fruit average accuracy ( $86 \%$ for
KNN and $84.4 \%$ for TMWE), false positive rate of (12.6\% for KNN and $14.3 \%$ for TMWE) and miss rate $(15.6 \%$ for KNN and $16.2 \%$ for TMWE). In this study, the proposed TMWE classifier gave a relatively high accuracy at lower computation cost. The earlier study shows that HOG descriptors outperformed the wavelet and PCA-SIFT. Image equalization can be skipped by using HOG Descriptor computation.

Zhang et al. [3] proposed a fine-grained classification of banana's ripening stages by using novel convolutional neural network architecture (CNN). CNN classification for banana ripening was firstly introduced in this paper. There were certain advantages to this work. First, it automatically extracts the multi-scale feature (global and local) of the banana images in the training process. These features were used for classifying different maturity stages of banana. 17,312 images were used for experimenting the process, 30 images were captured every day for 14 days and the CNN architecture produced had a classification accuracy of $94.4 \%$. Second, they also used defected bananas for classification experiments. Twenty batches of bananas, 197 with similar size, color, and weight (200-245) were selected for the experiment and before experimenting they were sanitized with $1 \% \mathrm{NaOCl}$ for 15 min, then rinsed with water and dried at ambient temperature. During the experimenting process, they were stored inside a stability chamber for 14 days under conditions of darkness at $25{ }^{\circ} \mathrm{C}$ and $75 \%$ of relative humidity. Images were captured (PNG format) using a Digital SLR camera with fluorescent light conditions. The white balance was set to the white fluorescent light and the images $(3200 * 2400$ pixels) were captured with a white background. Each image was represented in sRGB (standard RGB) color space.

Each image was resized into $256 \times 256$ and they manually labeled the images into 7 categories, and 14 categories according to the date of capturing. Among them, fifty percent of the images were taken as a training dataset, $30 \%$ for the training process and $20 \%$ for the testing process. In the training process, a backpropagation mechanism was used to eliminate noises. $\mathrm{CNN}$ was used to predict the probability of classification of input banana image and it was composed of a convolutional layer with a rectified linear unit (ReLU), max pooling and fully connected layer with ReLU. Positive images (at the same ripening stage as the original image), negative images (not at the same ripening stage as the original image), the original image and the label of the original images were jointly inputted into the $\mathrm{CNN}$ structure. There were 3 convolution layers. In convolution layer 1 (48 kernels of size $3 \times 7 \times 7)$ and in convolution layer 2 (128kernels of size $3 \times 5 \times 5)$ kernels with above-mentioned size were applied to the input banana image in each layer combined with ReLU and a max pooling layer came after each layer. Convolution layer 3 (128 kernels of size $3 \times 3 \times 3$ ) was also applied to the input banana image in the third layer combined with the ReLU. In a fully connected layer, 512 neurons combined with ReLU was used to perform neural network classification. Structured features were extracted from the proposed CNN framework. One softmax loss operation located at the end of the CNN channel was used to detect classification loss. Moreover, the triplet loss was also presented in this $\mathrm{CNN}$ architecture.

In the proposed model they got an accuracy of $94.4 \%$ for 7 categories classification after 31 iterations and training loss of this method was reduced to 0.15 as compared to other methods like Gabor + SVM, Wavelet + SVM, and Wavelet + Gabor + SVM. Meanwhile for 14 category classification accuracy of $92.4 \%$ was obtained after 34 iterations and training loss decreases to 0.22 . 
UluiSik et al. [4] proposed a technique to estimate the volume of tomato, which was captured using high-resolution cameras. Five different images of tomato, a variety grown in Turkey, were captured. The volume of the captured tomato fruits was computed by estimating horizontal and vertical distance by considering the ellipsoid shape. The main objective of this paper was to determine the quality of the tomato fruits in a faster and cheaper way to make it ready for packaging. Each tomato images $(1280 \times 960$ pixels) were captured inside a white box $(40 \times 40 \times 40 \mathrm{~cm})$, where each camera was attached to each side (centrally) of the box which was connected to PC using USB communication port. Each tomato was placed at the center of the camera's field of view and sufficient light source was provided using $50 \mathrm{~W}$ led light

Captured RGB color images of tomatoes were saved in jpg format. After capturing the images these were undergoing segmentation using simple thresholding method combined with morphological operations like dilation and filling. Segmentation method is used to segment the tomato from its background. To calculate the volume estimation, firstly the RGB color images with a white background were converted to gray scale images and then it was converted to a binary image using Otsu thresholding technique. After removing noises, edge detection techniques like Sobel, Canny were used to determine the outline of the tomato from its background. Among the edge detection algorithms, Canny edge detection method outperformed Sobel operator. Using regionprops function major and minor axis of each image was estimated and compared it with Euclidian distance of vertical and horizontal distances of tomato images. This function calculate Major axis Length, Minor axis length, centroid and surface area of the image. Binary images were divided into a small set of images which includes a set of cylindrical objects and the volume and surface area were calculated. The result of this image processing calculation was compared with the volume estimated by the ellipsoid formula. In this project, they made an assumption that tomato had an axisymmetric geometry.

The accuracy of the image processing method was reduced due to observed shadow at the bottom of the tomato during capturing and another reason for the volume differences was concavity shape of the tomato. Observed an estimation error under $20 \%$. Can improve the volume estimation by using machine learning algorithms like Artificial Neural Networks (ANNs), Support Vector Machines and k-Nearest Neighbor (k-NN).

Nowadays designing projects on production and post-harvest handling of fruits and vegetables had become an important subject for research scholars around the world. Quality assessment is the main issue arising in post-harvesting of fruits and vegetables. Kumar et al. [5] designed and implemented a vision based machine learning system for quality assessment. A custom-made image acquisition system is used for capturing sample images of pomegranate fruits. The spatial domain feature set and wavelet feature set were extracted from the sample images and using these features they trained the system by the help of both Support Vector Machines (SVM) and Artificial Neural Networks (ANNs). The aim of the project was to design a machine vision system that classifies the pomegranate into two classes, viz. diseased and healthy. Moreover, they continue the experiment to identify the best features and best classifier. The images were captured using a custom made image acquisition system under the presence of a light source. 60 diseased fruits and 60 healthy fruits were used for the experiment. Due to the variance in the light source, they equalized the image intensity values using histogram equalization method. Moreover, the use of histogram equalization reduces the quality of the image. To overcome this, they used a wavelet denoising to the histogram-equalized images.

The preprocessed images were then separated into spatial domain features (image region features, cloud features, Gray level co-occurrences, and texture features). ANNs outperformed SVM by using both spatial domain features and wavelet features for classifying pomegranate images. Although there is an increased accuracy by using wavelet features. Additionally, the standard deviation was less in ANNs due to the feature of multi-resolution analysis of images in wavelet transforms. SVM achieved an accuracy of $76.483 \%$ ANN got 81.97 using spatial features. By using wavelet features SVM got an accuracy of $80 \%$ and ANN had 92.65\%. 256 wavelet features were extracted in this method. Only two cultivators were used for the analysis.

Taofik et al. [6] developed a ripeness, detection system which adequates to defect 4 levels of [unripe 1, unripe 2, medium and ripe] ripeness of tomatoes and chilies. They experimented the system in each stage and the preliminary results were partially carried on between each stage and refined by PC, In spite of the fact that if the developed system was static, it is not possible to detect fruit color when the fruit is still on the tree. Moreover, if the fruit ripening system uses mobile technology rather than static PC and can detect ripeness of several types of fruits and this harvest prediction then it would be a more useful system for harvesting.

They captured the image using a smartphone camera. They chose tomato and chilly because the color features can be extracted very easily to detect ripening fruits whereas some other fruits can only be detected considering hardness as well. Noise can be reduced through preprocessing by using a median filter and thresholding. Before moving to test the maturity, they undergo segmentation step to determine the type of the fruit by k-means clustering method. Then the maturity was detected by fuzzy logic. Before applying the fuzzy process the concept of the control system was used in the images. The data collected at 65 days, 75 days, and 90 days of planting. The success level of testing data of tomato by $80 \%$ and for chilly was $90 \%$. The total accuracy from both was $85 \%$. Less amount of testing data were used to improve accuracy.

Farjana Sultana Mim et al. [7] implemented a method for classifying the mangoes into six maturity levels. More than 100 mangoes of different stages were used for the experiment. A total of 100 healthy mangoes, Himsagar mangoes, with uniform size, shape, and color were used for experimenting. Images of resolution 500x1000 were captured between 9 AM to 2 PM using a digital camera. Each image had a white background for easy segmentation. The background was removed to find the shape of the mango in the preprocessing stage. For this process at first red, green, and blue channels were separated. After converting the original image into a binary image segmentation was done on each image by using intensity dissimilarities in different regions. After background elimination, according to RGB and HSI models, a total of 24 image features were extracted for the categorization process. To select best feature sets they chosen an attribute selection filter from the datasets. Correlation-based attribute evaluator and best first search were used in the selection filter. By considering the individual predictive ability of each feature along with the degree of redundancy between them the correlation based attribute evaluator evaluates the subset of attributes. Correlation-based and information gain based 
evaluation has been used for finding the best feature sets. The best first search function explores the space of attribute subsets. This attribute selection method only stores four attributes $\mathrm{H}$-mean (mean of hue value), $\mathrm{H}$-median (median of hue value), H-LV (lowest value of hue) and I-LV (lowest value of intensity). More information was evaluated using Information gain and for this process, the ranker search method was used to rank the attributes in accordance with their information gain.

H-median (median of hue values), H-mean (mean of hue values), R-median (median of red values), R-HV (highest values of the red channel), R-mean (mean of red values), Smedian (median of saturation values), S-mean (mean of saturation values) features were extracted by using the above mentioned evaluation method. Among these 4 top-ranked features: H-median (median of hue values), H-mean (mean of hue values), R-median (median of red values) and R-HV (highest values of the red channel), were kept for classification. Two classification measurement using J48 decision tree (Weka version of C4.5 algorithm) was performed by using these attributes. 10 folds cross- validation technique was used to break data into 10 sets for describing training and testing data. A percentage split technique was also used. Using features extracted by correlation based attribute evaluator with Best-First search the decision tree provides $80 \%$ accuracy for the test set and by using information gain with Ranker search they got an accuracy of $72 \%$. The decision tree was tested by combining some selected features and accuracy was improved. Using H-mean, $\mathrm{H}$-median, R-median, and $\mathrm{H}-\mathrm{LV}$ it provides $88 \%$ accuracy and $96 \%$ accuracy by using H-mean, H-median, R-mean, and H-LV. Some errors were caused by intensity dissimilarity while capturing the images. They also planned to collect a large amount of data for enhancing accuracy.

Luiz Fernando Santos Pereira et al. [8] proposed a method for ripening of the papaya fruit using digital imaging and random forests. A sequence of physical/chemical analyses was accomplished and true maturity stage was obtained from pulp firmness measurements. Imaging and image analysis provided twenty-one hand-crafted color features computed from the peel and random decision forests were executed to forecast the ripening stage. More specifically, 57 golden papaya fruits were obtained and a total of 114 samples were utilized for the experiments. All samples were categorized into three stages of maturity. Images were captured using a digital camera which placed vertically at a distance of $17.5 \mathrm{~cm}$. The object was placed inside a box with matt black internal walls to ignore specular reflections and a 100W LED used as a light source.

Each of the color images were pre-processed with the region of interest (ROI) detection and normalization. The RGB, HSV, and CIE L*a*b* color spaces were extracted using MATLAB R2015a. Other important features were created by normalization and area computation. ROI selection was performed with thresholding in order to obtain important features from the color channel. From the binary image acquired, the fruit region was used to remove noise and for feature extraction $(\mathrm{Rm}, \mathrm{Gm}, \mathrm{Bm}, \mathrm{Hm}, \mathrm{Sm}, \mathrm{Vm}, \mathrm{L} *$, a *, and $\mathrm{b} *$ ). Random forests with two datasets (cross-validation and prediction set) are employed for the experiments. The crossvalidation set was applied to induce the models toward adjusting the hyper parameters (30 iterations), while the prediction test set was used to predict classification performance. This division was made in order to reduce the risks of over fitting by means of the Kennard Stone algorithm. Concerning all image features, $94.3 \%$ classification performance is obtained over the cross-validation set. The prediction set obtained $94.7 \%$ misclassifying only a single sample. For the group comparisons, the normalized mean of the RGB (red, green, blue) color space achieved better performance $(78.1 \%)$. Higher accuracy can be earned by extracting more features and deep learning techniques.

ShuqinTu et al. [9] developed a method for detecting different growth stages of the passion fruits and for identification of maturity using natural outdoor RGB-D images. Passion fruits on the same branch have different maturity stages. Then identifying maturity stages of passion fruit is a difficult process. Overall 400 images were captured using the Kinect camera, which was affixed on a tripod with telescopic adjustment. RGB images had the resolution $1024 \times 1248$ pixels and depth images had resolution 540x421 pixels and both these images were stored in JPG format. The images were captured between 3:00 PM and 6:00 PM. Because the images were affected by various natural conditions like sunlight, wind, and cloud. Moreover, by this fact, the Kinect sensor cannot be used in sunlight condition. The images were captured in the shady areas. Trellis-grown vines of passion fruit gave sufficient shadows for the image. To remove the noise inference and to improve the local contrast certain preprocessing method like adaptive histogram equalization and the median filtering were applied to the depth images. Randomly chosen training set and test set were used for maturity detection.

The captured images were classified into five categories based on the maturity stages; young $(\mathrm{Y})$, near-mature $(\mathrm{NM})$, nearyoung $(\mathrm{NY})$, mature $(\mathrm{M})$ and after-mature (AM). Machine vision algorithm involved two stages for detecting and classifying the passion fruits. First, a faster region based convolution neural networks (Faster R-CNN) was used for detecting passion fruits by using color and depth images which improves the detection performance. Faster-R-CNN enables the system to avoid the influence of illumination changes. Color images were used for maturity classification because depth images do not provide relevant information for maturity identification. Second, dense scale-invariant features transform (DSIFT) algorithm combined with localityconstrained linear coding (LLC) was used to extract the color features from each detected fruit regions. In addition, the color features extracted from the fruit regions, the RGB-DLLC were used to identify the maturity of fruits by using linear support vector machine (SVM) classifier. By using this approach they achieved $92.71 \%$ detection accuracy and $91.5 \%$ maturity classification accuracy.

Peng Wan et al. [10] developed a color analysis method for calculating the feature color values for detecting the maturity levels (green, orange and red) of fresh market tomatoes and then use backpropagation neural network (BPNN) classification technique to sort tomatoes based on the color features with potential future application in in-field yield estimation. With the help of computer vision technology, 200 samples per variety [Roma, Pear] was chopped randomly based on maturity in the lab. Among these 150 samples were captured for each variety. The images were captured under fluorescent light sources and the distance between the lens of the camera and the object was adjusted to $49 \mathrm{~cm}$. The objects were placed on black cloth for getting a black background. In the next process, preprocessing, tomato areas were segmented from the background using some image processing algorithm such as threshold segmentation, noise cancellation, image contour extraction, and boundary fill algorithm. For extra RGB values of tomato sample, an equal-area dividing method 
was used to divide the surface area of the tomato samples into five regions using Feature Color Area (FCA) and then convert the RGB color values into HSI color values. Before this process, the centroid of the tomato images was calculated to determine the contour of the tomato images.

Maturity levels of the tomato sampled were classified into three maturity levels namely Red Tomato (RT), a Mature Tomato (MT) and a Green Tomato (GT) using BPNN. The detection values for each of them were 1,2 and 3 respectively. BPNN consist of three layers. Input layer consist of five neutrons (feature color values) and the output layer had 1 layer where the output signals were 1(RT), 2(MT) and 3 (GT). The neuron number of the hidden layer was affirmed corresponding to the accuracy of the test results by using MATLAB 2016a. By using the 10 hidden layers they got an overall highest accuracy rate. $100 \%$ accuracy for both red and mature tomato and for green tomato samples they got an accuracy of $97.92 \%$. The average accuracy rate of tomato samples was $99.31 \%$ and the standard deviation is $1.2 \%$.

\section{COMPARISON}

A comparative evaluation of different works included in this study is depicted in Table 1.

Table 1: Summary of Related Works

\begin{tabular}{|c|c|c|c|}
\hline & Publications & Method & Remarks \\
\hline 1 & Wu, Jingui, et al [1] & $\begin{array}{l}\text { RGB features were extracted. } \\
\text { A novel automatic algorithm with a weighted } \\
\text { vector machine and bi-layer classifier. }\end{array}$ & $\begin{array}{c}\text { Recognition time is quit long with vast } \\
\text { amount of data. }\end{array}$ \\
\hline 2 & Tan, Kezhu, et al. [2] & $\begin{array}{l}\text { HOG features and color features were } \\
\text { extracted. } \\
\text { KNN (K-Nearest Neighbor) and TMWE } \\
\text { (Template Matching with Weighted Euclidean } \\
\text { Distance) classifiers. }\end{array}$ & $\begin{array}{l}\text { Image equalization can be skipped by using } \\
\text { HOG Descriptor computation. High accuracy } \\
\text { achieved at lower computation cost. }\end{array}$ \\
\hline 3 & Zhang, Yan, et al. [3] & $\begin{array}{l}\text { Multi-scale features were extracted. } \\
\text { Convolution Neural Network (composed of } \\
\text { convolution layer and Rectified linear unit } \\
\text { (ReLU)) used to classify banana, Max pool } \\
\text { and soft max were used to calculate errors. }\end{array}$ & $\begin{array}{l}\text { They determined the training loss in order to } \\
\text { increase the accuracy by increasing the } \\
\text { iterations. }\end{array}$ \\
\hline 4 & $\begin{array}{l}\text { UluiŞik, Selman, } \\
\text { FikretYildiz, and } \\
\text { AhmetTuranÖzdemİr. } \\
\text { [4] }\end{array}$ & $\begin{array}{c}\text { RGB images. } \\
\text { Segmented using Otsu thresholding combined } \\
\text { with dilation and filling. } \\
\text { Edge detection algorithms and region } \\
\text { properties. }\end{array}$ & $\begin{array}{l}\text { Calculated the volume of the tomato and } \\
\text { result of image processing is compared with } \\
\text { ellipsoid formula. Different shapes of the } \\
\text { images caused the image processing system } \\
\text { to determine the volume. }\end{array}$ \\
\hline 5 & $\begin{array}{l}\text { Kumar, Arun, Vijay S. } \\
\text { Rajpurohit, and Bhairu } \\
\text { J. Jirage. [5] }\end{array}$ & $\begin{array}{l}\text { Spatial domain features and wavelet features } \\
\text { were extracted for training. } \\
\text { SVM and ANNs is used for classification. }\end{array}$ & $\begin{array}{l}\text { ANNs outperformed SVM by using both } \\
\text { spatial domain features and wavelet features }\end{array}$ \\
\hline 6 & Taofik, A., et al. [6] & $\begin{array}{l}\text { Color features were extracted. } \\
\text { Noise cancelled using median filter and } \\
\text { thresholding. } \\
\text { K-means is used for classification. } \\
\text { Ripeness was detected by fuzzy logic. }\end{array}$ & $\begin{array}{l}\text { While using less amount of sample they got } \\
\text { better accuracy. In order to classify chilly } \\
\text { and tomato more than color features they } \\
\text { calculated the hardness. The developed } \\
\text { system cannot be used for harvesting the } \\
\text { objects from tree. }\end{array}$ \\
\hline 7 & $\begin{array}{l}\text { Mim, Farjana Sultana, } \\
\text { et al. [7] }\end{array}$ & $\begin{array}{l}\text { RGB and HSI features were extracted ( } 24 \\
\text { features). } \\
\text { Correlation based and information gain based } \\
\text { evaluation has been used for finding the best } \\
\text { feature sets. } \\
\text { Decision tree was used for classification. }\end{array}$ & $\begin{array}{c}\text { Small amount of data was collected for } \\
\text { training and extracted a large number of } \\
\text { features were extracted. Better results have } \\
\text { been obtained. }\end{array}$ \\
\hline 8 & $\begin{array}{l}\text { Pereira, Luiz Fernando } \\
\quad \text { Santos, et al. [8] }\end{array}$ & $\begin{array}{l}\text { RGB, HSV and CIE L*a*b* color spaces were } \\
\text { extracted. } \\
\text { Important features were extracted using region } \\
\text { of interest (ROI) detection and normalization. } \\
\text { Random Forest method used for prediction. }\end{array}$ & $\begin{array}{l}\text { They extracted a large number of features } \\
\text { and among them the best features selected by } \\
\text { using region of interest selection. By using } \\
\text { these features they got better performance. }\end{array}$ \\
\hline 9 & Tu, Shuqin, et al. [9] & Faster region based convolution neural & $\begin{array}{l}\text { In order to get better result they also captured } \\
\text { the depth of the image and got a better result }\end{array}$ \\
\hline
\end{tabular}




\begin{tabular}{|c|c|c|c|}
\hline & & $\begin{array}{l}\text { networks (Faster R-CNN) used for detection. } \\
\text { For extracting color features, dense scale } \\
\text { invariant features transform (DSIFT) } \\
\text { algorithm combined with locality-constrained } \\
\text { linear coding (LLC) was used } \\
\text { The maturity of fruits by using linear support } \\
\text { Vector machine (SVM) classifier. }\end{array}$ & $\begin{array}{c}\text { even though they used a large amount of } \\
\text { sample images. }\end{array}$ \\
\hline 10 & Wan, Peng, et al. [10] & $\begin{array}{l}\text { For segmentation algorithm such as threshold } \\
\text { segmentation, noise cancellation, image } \\
\text { contour extraction and boundary fill algorithm } \\
\text { were used. } \\
\text { RGB and HIS color space. } \\
\text { Sampled the images into regions by Feature } \\
\text { Color Area (FCA). } \\
\text { Back Propagation Neural Network (BPNN) } \\
\text { for classification. }\end{array}$ & $\begin{array}{l}\text { This method got highest accuracy when } \\
\text { compared with other works in this study, } \\
\text { even though it used } 10 \text { hidden layers. }\end{array}$ \\
\hline
\end{tabular}

\section{CONCLUSION}

There exists a bunch of methods and techniques in image analysis to detect maturity levels of fruit images and thereby estimating the accuracy levels of ripening of each image. We investigated the relative efficiency and detection power of state-of-the-art methods found in the literature. It is generally observed that the accuracy is severely affected due to variation in intensity values, complex backgrounds, and shadows. Also, almost all the methods required a lot of computational time to analyze the images. The number of training samples also varied from method to method. The scope of using deep learning for maturity detection may be treated as a promising direction for further research.

\section{REFERENCES}

[1] Wu, Jingui, et al. "Automatic Recognition of Ripening Tomatoes by Combining Multi-Feature Fusion with a BiLayer Classification Strategy for Harvesting Robots." Sensors 19.3 (2019): 612.

[2] Tan, Kezhu, et al. "Recognising blueberry fruit of different maturity using histogram oriented gradients and colour features in outdoor scenes." Biosystems Engineering 176 (2018): 59-72.

[3] Zhang, Yan, et al. "Deep indicator for fine-grained classification of banana's ripening stages." EURASIP Journal on Image and Video Processing 2018.1 (2018): 46.

[4] UluiŞik, Selman, FikretYildiz, and vision system for tomato volume estimation." 2018
Electric Electronics, Computer Science, Biomedical Engineerings' Meeting (EBBT). IEEE, 2018.

[5] Kumar, Arun, Vijay S. Rajpurohit, and Bhairu J. Jirage. "Pomegranate fruit quality assessment using machine intelligence and wavelet features." Journal of Horticultural Research 26.1 (2018): 53-60.

[6] Taofik, A., et al. "Design of Smart System to Detect Ripeness of Tomato and Chili with New Approach in Data Acquisition." IOP Conference Series: Materials Science and Engineering. Vol. 288. No. 1. IOP Publishing, 2018..

[7] Mim, Farjana Sultana, et al. "Automatic detection of mango ripening stages-An application of information technology to botany." ScientiaHorticulturae 237 (2018): 156-163.

[8] Pereira, Luiz Fernando Santos, et al. "Predicting the ripening of papaya fruit with digital imaging and random forests." Computers and Electronics in Agriculture 145 (2018): 76-82.

[9] Tu, Shuqin, et al. "Detection of passion fruits and maturity classification using Red-Green-Blue Depth images." Biosystems Engineering 175 (2018): 156-167.

[10] Wan, Peng, et al. "A methodology for fresh tomato maturity detection using computer vision." Computers and electronics in agriculture 146 (2018): 43-50.

[11] Li, Bairong, Yan Long, and Huaibo Song. "Detection of green apples in natural scenes based on saliency theory and Gaussian curve fitting." International Journal of Agricultural and Biological Engineering 11.1 (2018): 192-198. 